\title{
Lunar Prospecting: Searching for Volatiles at the South Pole
}

\author{
Jay Trimble ${ }^{1}$ and Robert Carvalho ${ }^{2}$ \\ NASA Ames Research Center, Mountain View, CA, 94035, USA
}

\begin{abstract}
The Resource Prospector is an in-situ resource utilization (ISRU) technology demonstration mission, planned for a 2021 launch to search for and analyze volatiles at the Lunar South Pole. The mission poses unique operational challenges. Operating at the Lunar South Pole requires navigating a surface with lighting, shadow and regolith characteristics unlike those of previous missions. The short round trip communications time enables reactive surface operations for science and engineering. Navigation of permanently shadowed regions with a solar powered rover creates risks, including power and thermal management, and requires constant real time decision making for safe entry, path selection and egress. The mission plan requires a faster rover egress from the lander than any previous NASA rover mission.
\end{abstract}

\section{Introduction}

$\mathrm{T}$ he RP Mission is a Lunar exploration mission, driven by science goals, but differentiated from a pure science mission by its resource utilization focus. RP is the next step in the characterization of surface volatiles for utilization for future missions to the Moon and Mars. To locate, characterize and analyze volatiles, the mission includes a rover for mobility, prospecting instruments to locate and characterize volatiles, a drill to collect regolith samples, and an in-situ resource utilization (ISRU) payload for analysis. The surface mission is short, the environment has many unknowns, and operational constraints create unique challenges.

\section{Mission Overview}

Strategic Knowledge Gaps (SKGs) are gaps in what we need to know to plan future space exploration missions. RP is designed to address gaps needed to plan human missions to the Moon before going to Mars. Returning to the Moon allows for testing technologies closer to home than Mars Missions, and allows for the possibility of using lunar resources to reduce the cost of solar system exploration. Previous Lunar Missions have identified volatiles at the

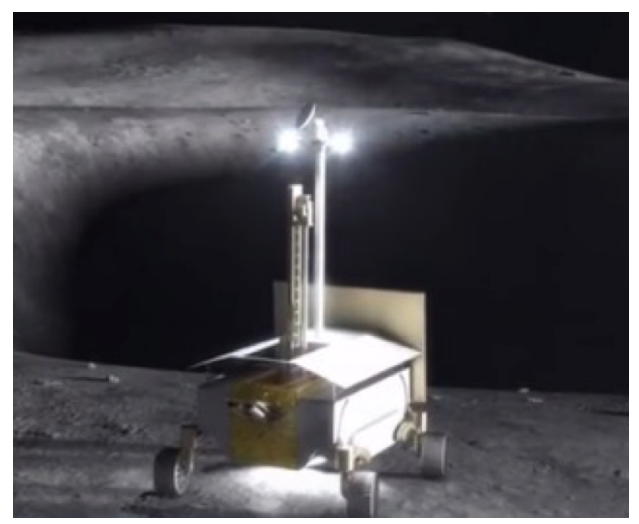

Figure 1 - Artists concept of the RP Rover on the Lunar Surface
Lunar Poles, however the scope, scale and extent are unkown, and await a surface mission to further our knowledge. Here are some specific knowledge gaps identified by the Lunar Exploration Analysis Group (LEAG):

Determine

-... the physiography and accessibility of cold traps

-... the concentration of volatile species within 1-2 m of the surface

-... the variability of water concentration on spatial scales of

10 's of meters

-... the mineralogical, elemental, molecular, isotopic makeup of the volatiles

-... the lunar surface trafficability

\footnotetext{
${ }^{1}$ Mission Operations Manager, Intelligent Systems Division, Mail Stop 269-3, AIAA Member Grade for first author.

${ }^{2}$ Systems Engineer, Intelligent Systems Division, Mail Stop 269-1
}

American Institute of Aeronautics and Astronautics 
The only place where lunar volatiles have been conclusively demonstrated is at the Lunar Crater Observation and

\section{Prospecting}

Neutron Spectrometer System (NSS)

- Water-equivalent hydrogen $>0.5$

wt $\%$ down to 1 meter depth

NIR Volatiles Spectrometer

System (NIRVSS)

- Surface H2O/OH identification

- Near-subsurface sample

characterization

- Drill site imaging

- Drill site temperatures

Figure 2 - Prospecting Instrument Overview Sensing Satellite (LCROSS) impact site. Other permanently shadowed regions (PSRs) may contain volatiles, and temperature models and Lunar Reconaisance Orbiter (LRO) data suggest volatiles may also exist near the surface in areas lit at low angles for a short time each month. But the spatial distribution of lunar volatiles is unclear. Therefore some form of prospecting is required, that is, the ability to move, to sense volatiles under the surface, and to access them. ${ }^{1}$

RP consists of a rover that provides mobility for a payload with prospecting instruments for identification of surface and sub-surface volatiles, a drill for sample collection, and an ISRU payload for extraction and analysis of volatiles. The prospecting instruments consist of a neutron spectrometer capable of detecting hydrogen-bearing compounds to a depth of 1 meter and a near-infrared spectrometer capable of detecting water and $\mathrm{OH}$ bound to minerals on the surface and brought to the surface by the drill. The ISRU payload consists of the Oxygen and Volatile Extraction Node (OVEN) and Lunar Advanced Volatile Analysis (LAVA). The drill can bring up material from up to $1 \mathrm{~m}$ in depth and place samples in a crucible for transfer to a sealed oven for heating. OVEN can heat samples to $150 \mathrm{C}$ for driving volatiles out of the samples and into a gas chromatograph and can further heat regolith samples to $900 \mathrm{C}$ to produce water vapor via the process of hydrogen reduction. ${ }^{8}$

The rover will operate on the lunar surface for seven to ten days at a location where both sun exposure and direct to Earth (DTE) communication conditions overlap, and it will be teleoperated from Earth using the Deep Space Network (DSN). The rover will be capable of entering shadow and operating for up to six hours. At the end of ten days, the sun will go down, and operating solely on solar and battery power, it is not expected to survive the cold of the lunar night.

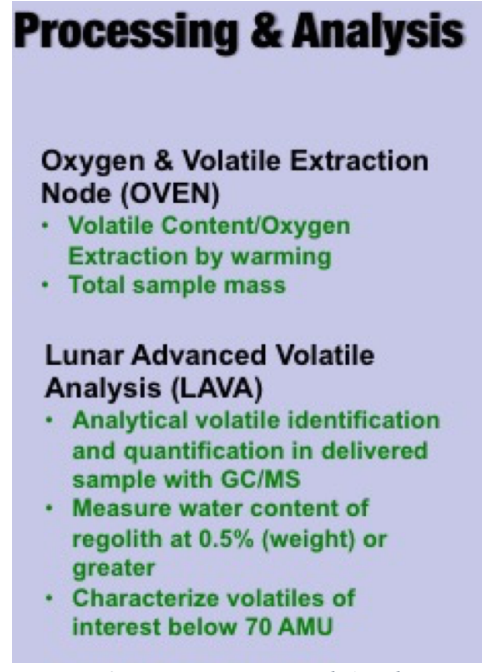

Figure 3 - Processing and Analysis Instrument Overview
The Mission Operations System (MOS) is responsible for executing the mission to meet the science and mission requirements levied by the project. Requirements are structured as minimum success, full success and extended. To meet minimum success criteria the rover/payload must take measurements from two places separated by at least 100 meters. Full success requires measurements from two places separated by 1000 meters and include surface and subsurface measurements, a sample acquisition from a shadowed area, and a demonstration of ISRU. Stretch goals require measurements in eight locations across 1000 meters point to point distance, with processing and analysis of subsurface material in at least four locations.

The nominal plan is to prospect across the planned traverse path (rails), with detailed exploration and analysis to take place at planned exploration sites (stations). When warranted based on prospecting data, science may request path deviations for further investigation using prospecting and/or ISRU instruments.

\section{A. Shadow Operations}

A primary mission objective is the exploration of one or more permanently shadowed regions (PSR). PSRs are among the coldest known areas in the solar system, and may not have been exposed to light for hundreds of millions of years. The operation in these unknown shadowed environments will present challenges. The regolith properties are not known. It is believed that the regolith could present driving hazards including a brittle surface structure which may unexpectedly collapse under the rover, unanticipated depths of loose regolith which could allow the wheels to sink in, unusual electrical charging of the regolith, and poorly understood surface traction. ${ }^{5}$ The rover is solar powered, limiting shadow operations to roughly six hours, including the non-permanent shadow area that may surround the PSR. The rover will need to provide its own light source for imaging. Thermal management, power management, entry and exit path selection, and use of the rovers sensors to select safe entry and exit paths constitute further operational challenges. The thermal management challenges also affect power management, which affects the safe duration for PSR operations. The complex interplay of real-time power and thermal management, path selection, and reactive prospecting data in a PSR will require constant real time decision management during all shadow operations.

American Institute of Aeronautics and Astronautics 
Mission rules require the rover battery to maintain a minimum twenty percent charge. Upon emergence from a PSR the rover may need to be stationary during a recharge period of up to 24 hours, during which volatile analysis of samples collected in a PSR may be performed.

The mission plan begins with lower risk activities, with PSRs coming later in the timeline. The entry plan will take place in small discrete increments, with safety evaluations at each point. The science return from PSRs is anticipated to be the highest of the mission.

\section{B. Parallel Operations}

The compressed mission timeline, driven by the short surface duration, requires parallel operations and compressed decision cycles to optimize productivity, and minimize non-productive surface time. Non-productive time is any time spent not prospecting, collecting or processing. Ground decision times and charging times are key factors in nonproductive time. The operational approach will maximize productive surface time with a three-tiered process (reactive, tactical, strategic) and through parallel operations. Instead of a standard flow of instrument calibration and checkout followed by a handover to science (serial operations) we will be conducting science operations during calibration and checkout, meaning, we will be taking data as part of the exploration science in parallel with calibration and checkout. To further maximum surface time utilization, instrument operational readiness checks are planned to be performed during the cruise to the moon.

\section{Egress}

Recent planetary rover history shows that egress is a challenging phase of a rover's mission and the actual duration may vary from the planned duration. The challenges come from both nominal operations, such as deployments and checkouts of rover systems on the lander, but also from ambiguity of data for safe egress. RP is planned to have two egress ramps, and a safe path will need to be selected. The system is being designed to support rover egress from the lander in six hours. This is considerabily faster than previous NASA rover missions.

Spirit, on Mars, took twelve sols to get off the lander, her sister Opportunity took seven. Curiosity took its first drive sixteen sols after landing, however, there was no lander for Curiosity, as the sky crane placed the rover directly on the surface. Sojourner, in 1997, was the fastest, rolling down on to the Martian surface in under two sols. ${ }^{9}$

The RP six hour egress requirement imposes requirements on the lander design and on operational procedures. All possible checks and calibrations will be done during cruise phase, before landing. After landing, only those checkouts required for safe egress will be performed, and those will be done in parallel where possible. This requires power and communications margins. Non safety critical checks will continue post egress.

\section{Operations Overview}

Our operational focus for this paper is surface operations. The current surface operations plan is a hybrid of operational methods from human space-flight and from interplanetary rovers. The key parameters driving the operational design are:

- Near real-time command and control

- Variable and unpredictable communications latency on the scale that enables/impacts teleoperation capability

- Uncertainty about concentration of spatial distribution and volatiles that requires reactive operational response and adaptive traverse re-planning

- Short duration of surface operations

- Significant risk of losing DTE comm that would require rover movement to regain

- High levels of drill and sample-processing autonomy

\section{A. Decision Cycles: Round Trip Latency}

There is a degree of unpredictability in the round trip time from command uplink to verification. While the Earth to Moon round trip light time is short and predictable, the communications network and the Deep Space Network (DSN) introduce an unpredictable and variable latency. This is because the DSN is primarliy used for deep space missions with round trip times in minutes and hours, hence a few seconds is not meaningful. Data from the LCROSS and LADEE Lunar Missions missions indicates typical two- way signal transmission durations of 6 - 10 seconds with edge cases of 25 seconds and irregular bursts of 15 seconds. This is within the DSN performance range. For RP, the round trip latency is expected to be longer, ranging from 10-30 seconds. This is because of higher bandwidth

American Institute of Aeronautics and Astronautics 
requirements for RP. While short Earth-Moon distance presents the possibility of direct rover teleoperation, the latency and uncertainty introduced by the communications networks and the DSN make this infeasible.

Previous testing has shown that a rover can be teleoperated manually with a short round trip delay of up to ten seconds, though even at that delay operators preferred waypoint driving. ${ }^{2}$ Delays in the tens of seconds make direct teleoperation driving unsafe, as the rover may crash before the operator sees the crater it is falling into. ${ }^{6,7}$ Because of the larger delay, and the variable duration of the delay, a different approach to driving is required for RP. The current designed approach is with limited range waypoint commanding. ${ }^{10}$ The waypoint approach has been used on the MER missions with daily commanding of a series of waypoints, though RP will be operating with significantly less onboard autonomy and a much shorter planning cycle.

The rover driving approach will take advantage of the short overall latency, but not depend on direct real-time teleoperation. Waypoint selection and driving in near real-time enables significant flexibility in path selection and scientific responsiveness, and reduces the stress on the rover driver. ${ }^{2}$ Long command sequences and associated validations are not required. A command simulator is planned to quickly validate the short command uploads, showing that the rover will respond as expected to commands and avoid the observed hazards.

The current approach involves taking a stereo pair of images to a range of about eight meters, and then commanding a waypoint about half way across the field of view. At that point, another pair of images is taken, but while that is downloaded and processed, the next waypoint will be commanded to near the limit of verified safe terrain within the first image pair, where a third pair of images will be taken. When the second set of images is processed, the driver will then command a waypoint near the limit of the verified safe terrain within that pair of images, and the capture of a fourth set of images.

While the operations team will have the capability to react in real time to data from the prospecting instruments, the plan is to traverse along a pre-planned path, with set points for detailed exploration, sample acquisition and processing. That said, prospecting data may warrant changes of plan in real time.

\section{B. Prospecting and Science}

The nature of the science objectives, the instrument selection, and the sub-minute turnaround time provides the science team with greater flexibility in selecting targets than interplanetary surface missions. The approach for realtime science has been tested in previous payload field tests. ${ }^{3,4}$ It will allow the real-time scientist to react to data that is coming in from the spectrometers to determine where the rover goes next, much like a bloodhound follows a scent. In order to maximize the duration of the mission, this flexibility will be conducted within limited areas and timeframes at pre-defined science stations. In between the science stations, the rover will travel on a more fixed course. However, even within that, the real-time scientist would have the option to call for a halt and further investigation if they see the rover is driving over a spot that is significantly higher in water concentration than previous areas. As the mission progresses, and success goals are met, the real-time scientist will have more flexibility to call for deviations from the plan based on real time science data.

\section{Planning}

Planning refers to the activities necessary to develop and execute mission activities to accomplish mission objectives. Planning in this context does not include the definition of mission objectives, it includes the activities and steps to achieve mission objectives. Planning activities take place pre-mission and during the mission. Planning is in tactical and strategic cycles, with integration of data from real-time science. A mission plan typically includes spacecraft activities, i.e. all in space activities. Supporting documentation may also include ground and team activities.

\section{Science Stations and Rails}

Prior to launch, the science team will define areas of scientific interest based on orbital data, including hydrogen concentrations, permanent shadowing, elevation, and other data. These areas will form the basis for the traverse plan. Our plan is to define rails and science stations. Science stations are areas of detailed exploration, prospecting and analysis. Rails are traverses to get to stations. The mission plan calls for adhering to a pre-planned path while on rails, with deviations for safety.

The concept is to prospect along relatively fixed predetermined paths (rails) between the science stations, with adjustments made in near real-time for rover safety. The pre-determined paths are worked out within the plans, and will include margins for time and unexpected events. While on these rails, the driver will be focused on getting to the next science station.

American Institute of Aeronautics and Astronautics 


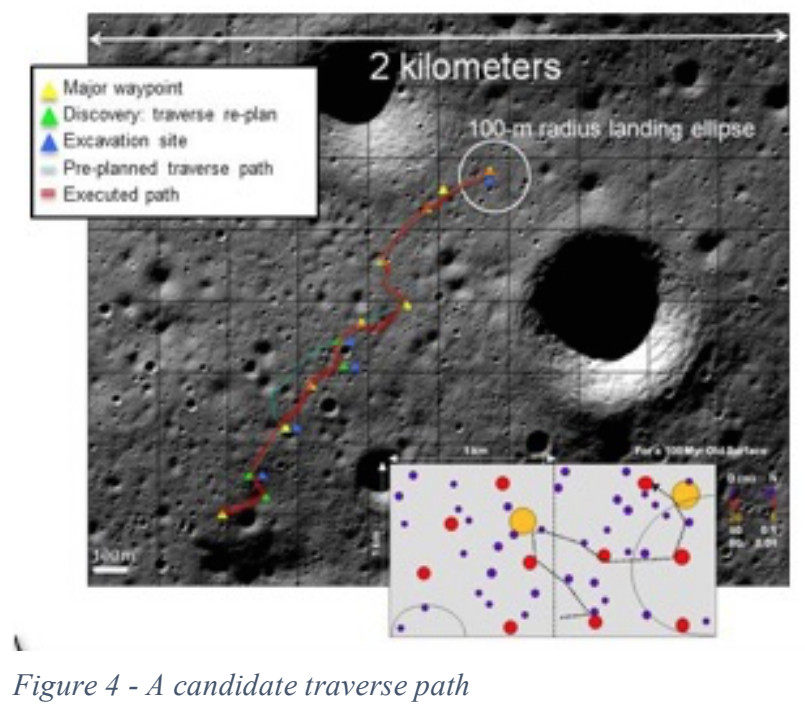

\section{E. Planning and Contraints}

NASA missions have well established processes for real-time decision making, as well as tactical and strategic planning. RP is building on these precedents. The combination of reactive decisions based on science and engineering data, the integration of a unique set of planning constraints particular to the RP environment and mission objectives, and a continuously operational asset create, in combination, requirements for new planning processes.

Unlike previous NASA Lunar Surface explorations with mobility, which were human missions, RP does not have extravehicular activity (EVA) constraint times, crew sleep times, or mobility range limitations based on safe proximity to an ascent vehicle. This enables options not available to the Apollo Lunar Explorations. It also creates no clear boundary between tactical and strategic planning. We have set the tactical shift at eight hours, based on the shift planning for the mission control team.

RP also operates with constraints that are unlike previous missions. These constraints include rapidly changing surface lighting conditions, a solar powered rover that must operate in light with the exception of short battery powered detours, reactive mission operations that make tactical and strategic planning subject to frequent changes, uknown surface characteristics, and a requirement for DTE communications. The operations team design consists of reactive and near-real time decision making, tactical and strategic planning.

The strategic plan encompasses the complete mission and includes all activities necessary to achieve full success criteria. It includes margin which allows for stretch goals (extended mission), mission safety, and reactive science opportunities. It will be updated on twelve hour shifts, with data from real-time and tactical operations. The strategic plan includes constraints which are not subject to change, such as lighting and DTE.

Tactical planning is based on a roughly eight hour timeframe. The planned approach for tactical planning is to continually update the planned route to the next science station, taking into consideration the choices made by the driver and real-time science at each step. The tactical planner is also the operations position intended to operate as the train conductor, alerting the driver and real-time science when it is time to leave a given area in order to arrive at the next station with the proper safety margin.

Prospecting is the nominal driving mode, with continuous data from the prospecting instruments. A real-time scientist, co-located with the rover driver and the rover co-driver are responsible for making real-time path decisions. While operations on the rails between the science stations are focused on getting to the next stations, the real-time science operator will continue to monitor the prospecting instruments. Should this operator see data which clearly calls out for further investigation, they can request a stop. Then they can ask for either a short mapping of the local resources, or even a sample collection. This would need to be done within the margins set up in the tactical and strategic plans. The real-time science position also gives updates to the tactical and strategic planners for incorporation into the mission plan.

\section{Distributed Operations and Team}

The development team for RP is distributed among multiple NASA Centers. Ames Research Center (ARC) leads the flight project office, mission system engineering, science, mission operations, and some instruments. Kennedy Space Center (KSC) leads development of the ISRU payload, working in collaboration with the Johnson Space Center (JSC). The lander design is led out of Marshall Space Flight Center (MSFC), working with JSC. The lander builder is still the subject of negotiation, with possible international and/or commercial partnerships.

For location of the mission operations team we looked at several options:

- A centralized mission control center

- Fully distributed mission control

- Hybrid distributed/centralized

American Institute of Aeronautics and Astronautics 
The centralized mission control option would have all mission control team members travel to one central location. We used KSC for costing purposes for the study. The fully distributed option had all operators operating from their home institution. The hybrid option looked at the need to have some operators co-located with others distributed.

The decision was based on an evaluation of parameters:

- Facilities cost

- Type of decisions and affect of distribution vs. co-location

- Training frequency and the cost of travel for training

- Cost of travel for mission operations

Facilities cost was thought to be a significant driver up front. The cost of facilities turned out to be low, relative to the cost of travel. The decisive factor was that training is more important than co-location. Our plan is to train weekly or bi-weekly during the last year before launch. The cost of operating out of a central location was driven by travel cost and the cost of the team members. For each training exercise out of a central location would come the overhead of travel before and after. This effectively almost doubles the cost of team member time and decreases our flexibility to plan training exercises as all would be forced onto to pre-planned travel schedules.

For the effect of co-location on operator decisions, our instincts gravitated towards previous experience which was to have critical real-time decision makers with dependencies in their decisions, co-located. Weighing towards flexibility, we noted the long history of distributed operations. We do not have conclusive data that would impact this decision. For real-time and near real-time operations, whether co-located or distributed, most decisions are made over voice loops, even for co-located operators. The capability to perform complex operations with distributed teams has been demonstrated.

The bottom line on the decision was this - training is more important than co-location. Thus, all operators are baselined to operate the mission from their home institution. In California, at ARC, will be the flight director, mission manager, science lead, mission system engineers, command and data flow engineers, rover drivers, science planners and prospecting instrument science leads. In Florida, at KSC, will be the payload ops lead, systems team and command positions. In Houston, at JSC, will be the rover systems team. The lander team definition is pending the determination of NASA's role with a prospective lander partner.

We leave open the possibility that if, based on experience in mission simulations, it is determined that distributed operations for select positions creates a threat to mission success, or a significant decrease in operational efficiency, that select positions could be co-located. Data from simulations conducted to date indicates that we can be efficient and effective in operations with a fully distributed team.

\section{Distributed Operations Test}

Because of partnering negotiations between NASA and other agencies and the advanced technology readiness level (TRL) of the payload, RP was given a unique opportunity to conduct a proof of concept for the mission during phase A, which helped to prove out several key concepts. This Distributed Operations Test (DOT) used a prototype rover, carrying an engineering test unit version of the payload. This surface segment prototype was developed, integrated, and tested in less than a year. It was further integrated with an early prototype of the Ground Data System (GDS), and operated in a distributed manner, similar to how the full mission will be operated.

American Institute of Aeronautics and Astronautics 


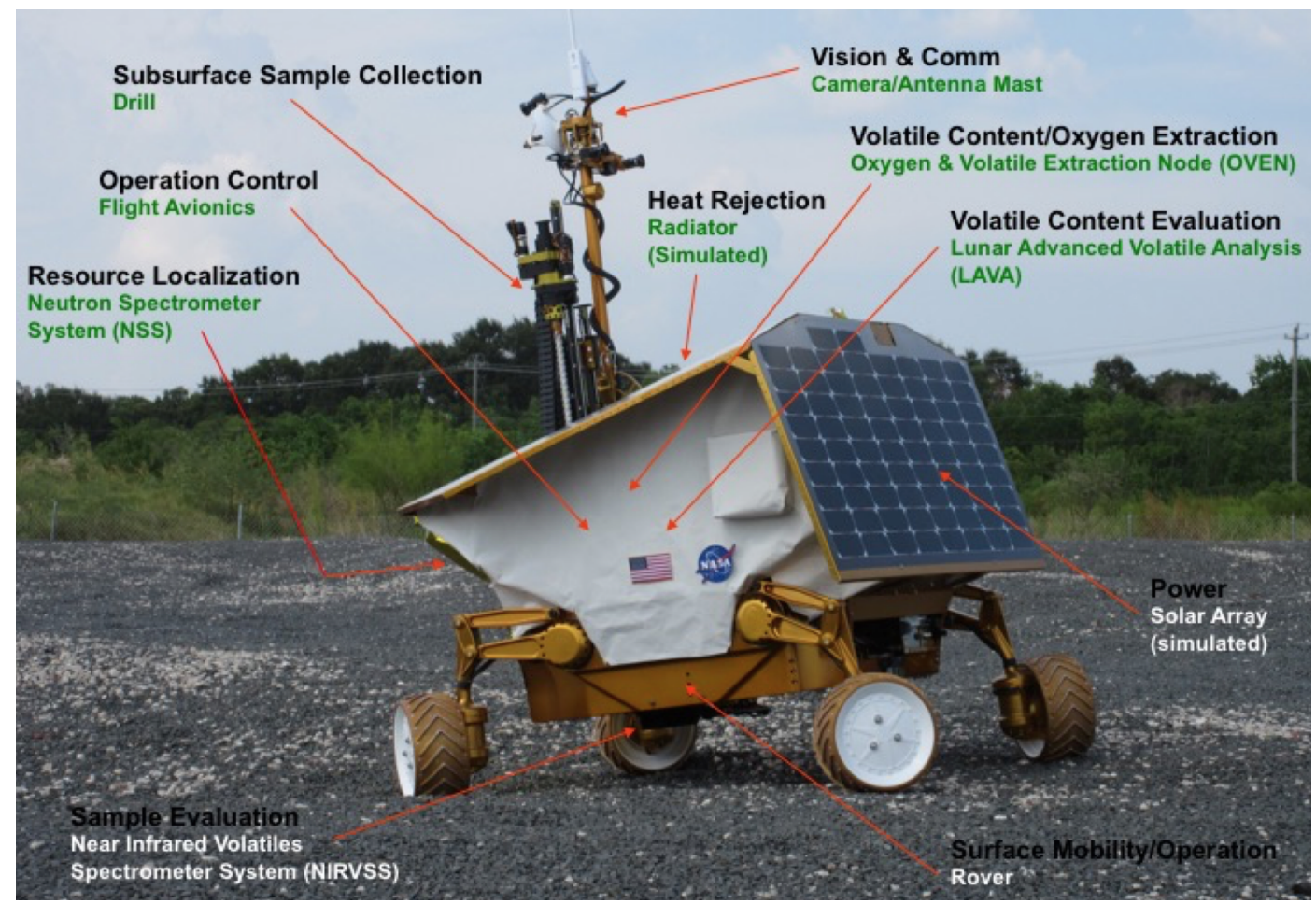

Figure 5 - RP-15 Rover and Payload

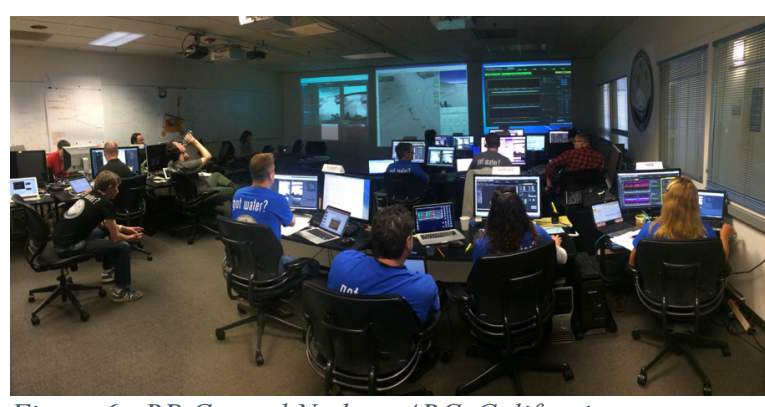

Figure 6 - RP Control Node at ARC, California
While the rover was operated in a test area at JSC, the DOT simulated many of the key activities of the surface mission for RP. This included waypoint driving, prospecting, some limited image-based navigation, tactical planning (though not re-planning), drilling, sample collection and processing. The DOT demonstrated the potential to operate the mission in the planned distributed manner, and also provided a range of lessons for improving the full mission.

The lessons learned from the DOT spanned a considerable range. One quarter of the lessons had to do with hardware and integration. These issues are understandable given the prototype nature and accelerated schedule for the DOT integration. Another quarter of the lessons had to do with configuration management, which is more challenging given the distributed nature of the team both in development and operations. Some of the more interesting lessons had to do with the workload experienced by team members during integration and operations. These workload lessons have led to a review of the planned staffing for the full mission and initiated the modeling of the roles and responsibilities discussed above. Other lessons had to do with the online approach for procedure distribution and tracking in operations, leading to a trade study to find a better approach to manage and track procedures within a distributed operations environment. 


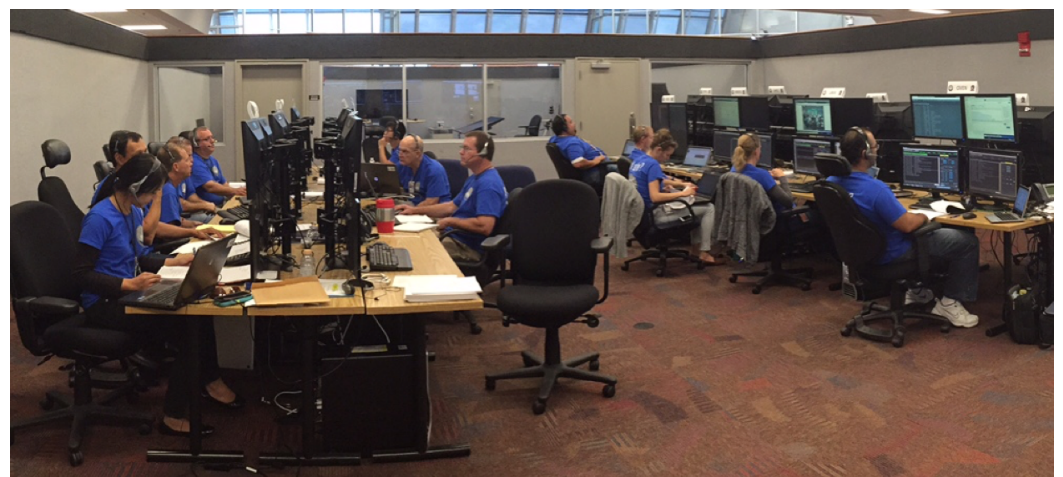

Figure 7 - RP Payload Control Node at KSC, Florida
Further testing in work this fiscal year includes dealing with the more difficult lighting conditions expected at the lunar poles, and integration of more advanced visual navigation tools. There will also be tests conducted to begin to prove the feasibility of the intended operational cycles and tempo for the mission, from real-time, through tactical, and on to strategic.

\section{Conclusion}

$\mathrm{RP}$ is in the formulative phase. The team has been able to validate key operational concepts in simulation, including distributed real-time operations, limited waypoint driving, and execution of the basic science and payload operations. Data from field tests supports the design of prospecting operations. Further pre-mission operations design challenges include design and validation of the mission planning cycles and integration with reactive decision making. The team will have a year before launch to train for operations, and will learn, where possible, on the flight hardware by shadowing testing. Lunar resources await characterization and discovery. The RP team is ready.

\section{Acknowledgments}

Dan Andrews, Tony Colaprete, Amanda Cook, Matt Deans, Rick Elphic, Sarah Hobart, Mark Shirley, Ryan Vaughn.

\section{References}

${ }^{1}$ Colaprete, A., Elphic, R.C., Andrews, D., Sanders, G., McGovern, A., Vaughan, R., Heldmann, J., and Trimble, J. "Resource Prospector: Mission goals, relevance, and site selection," Lunar Exploration Analysis Group Meeting, Columbia, MD, 2015.

${ }^{2}$ Gingras, David, "Deployment of the CSA Teleoperation Robotic Testbed 2 at Rougemont Sand Quarry, Test Report", Canadian Space Agency, 24 Mar 2014.

${ }^{3}$ Heldmann, J.L., Colaprete, A., Elphic, R.C., Mattes, G., Ennico, K., Fritzler, E., Marinova, M.M., McMurray, R., Morse, S., Roush, T.L., and Stoker, C.R. "Real-time science operations to support a lunar polar rover mission," Advances in Space Research Vol. 55, 2015, pp. 2427-2437.

${ }^{4}$ Heldmann, J.L., Colaprete, A., Elphic, R.C., Lim, D., Deans, M., Cook, A., Roush, T., Skok, J.R., Button, N.E., Karunatillake, S., Stoker, C., Marquez, J., Shirley, M., Kobayashi, L., Lees, D., Bresina, J., and Hunt, R. "Lunar polar rover science operations: Lessons learned and mission architecture implications derived from the Mojave Volatiles Prospector (MVP) terrestrial field campaign," Advances in Space Research, in review, 2016.

${ }^{5}$ Kring, David A. "Regolith properties of permanently shadowed regions (PSRs) in polar regions of the Moon and implications for rover trafficability: Outlining the problem“ SSERVI Polar Regolith Workshop, December 2015.

${ }^{6}$ Krotkov, E., Simmons, R., Cozman, F., \& Koenig, S. "Safeguarded teleoperation for lunar rovers: From human factors to field trials," IEEE Planetary Rover Technology and Systems Workshop, April 1996.

${ }^{7}$ McGovern, Douglas E. "Experiences and results in teleloperation of land vehicles," Sandia Report SAND90-0299, 1990.

${ }^{8}$ Quinn, J., Smith, J., Captain, J., Paz, A., Colaprete, A., Elphic, R., and Zacny, K. "Resource Prospector: The RESOLVE payload," Lunar Exploration Analysis Group Meeting, Columbia, MD, 2015. 
${ }^{9}$ Teitel, Amy S., “Compared To Past Mars Rovers, Curiosity Is Cruising” Motherboard, 27 Aug 2012.

${ }^{10}$ Wettergreen, D., Bualat, M., Christian, D., Schwehr, K., Thomas, H., Tucker, D., \& Zbinden, E. "Operating nomad during the Atacama Desert trek," Field and Service Robotics Springer London. January 1998, pp.82-89. 